\title{
An Internet of Intelligences with Geospatial Information Diffusion Model for Inferring Disasters in Information Isolated Islands
}

\author{
Chongfu Huang ${ }^{1,2}$

\begin{abstract}
${ }^{1}$ Key Laboratory of Environmental Change and Natural Disaster, Ministry of Education, Beijing Normal University, Beijing 100875, China;

${ }^{2}$ Academy of Disaster Risk Sciences, Faculty of Geographical Science, Beijing Normal University, Beijing 100875, China
\end{abstract} \\ Email: hcongfu@bnu.edu.cn
}

\begin{abstract}
An internet of intelligences constructed with "model-view-controller" frameworkis composed of six functional modules: data declaration, data review, data query, disaster assessment, user management, and system management. There are four user role permissions: data declarer, daily administrator, disaster relief administrator, system administrator. With the internet of intelligences embedded in the geospatial information diffusion model, it becomes possible for us to infer disasters in the information isolated islands in the disaster area, instead of disaster assessment by using a statistical formula based on historical disaster data. The new approach is not only the accuracy of the assessment can be improved, but also the disasters can be easily updated. We chose ThinkPHP5 to be the development framework. The logical structure of the system database and the tables are designed according to the principles of database design and paradigms. The geospatial information diffusion model is programmed in PHP language in the WAMP environment. Going back to the scene of the 2008 Wenchuan Earthquake and inferring the economic loss rate and population casualty rate and in the 10 severely affected areas, we know that the geospatial information diffusion model is more accurate than linear regression method and the random forest method.
\end{abstract}

Keywords: Internet of Intelligences, Geospatial, Information Diffusion, Disaster Assessment, Wenchuan Earthquake

\section{用地理空间信息扩散模型推测信息孤岛灾情的智联网}

\author{
黄崇福 ${ }^{1,2}$
}

\author{
${ }^{1}$ 北京师范大学，环境演变与自然灾害教育部重点实验室，北京 100875 , 中国 \\ ${ }^{3}$ 北京师范大学地理科学学部灾害风险科学研究院, 北京 100875 , 中国 \\ 电子邮箱: hchongfu@bnu. edu. cn
}

\section{摘要}

用 “模型-视图-控制器” 框架构建的智联网, 由数据申报、数据审核、数据查询、灾情评估、用户管理、系 统管理等六个功能模块组成，设置了数据申报员、日常管理员、救灾管理员、系统管理员等四种用户角色权 限。在嵌入的地理空间信息扩散模型支持下，能对灾区中的信息孤岛的灾情进行在线 “采点外推” ，解决了 根据历史数据统计公式 “隔空判灾” 的精度低，且不可在线更新的问题。我们选用 ThinkPHP5 框架作为开发 架构, 根据数据库设计原则和范式设计了系统数据库逻辑结构和数据库表，在 WAMP 环境下用 PHP 语言对空间 信息扩散模型进行编程。再现推测 2008 年汶川地震 10 个极重灾区人口死亡率和经济损失率，结果表明，地 理空间信息扩散模型比线性回归方法和随机森林方法更为准确。

关键字: 智联网, 地理空间, 信息扩散, 灾情推测, 汶川地震 


\section{1.引言}

进入二十一世纪, 自然环境和社会经济都在加 速变化。全球变暖, 导致与气候有关的灾害数量出 现惊人增长; 社会经济快速发展, 使得受灾地区的 灾情形态与过去明显不同。

以往, 重大自然灾害发生后, 人们习惯于用历 史灾害数据统计回归得来经验公式, 对灾情进行快 速评估 ${ }^{[1]}$ 。这种经验性的“隔空判灾”，能将灾情的 估计精度控制在数量级误差之内, 就已经很不错 ${ }^{[2] 。}$

为完成国家重点研发计划课题 “重大自然灾害 多层级精准救助关键技术研究” 中及时、精准而全 面地评估灾情的研究任务, 合理推测信息孤岛灾情, 地理空间信息扩散模型于 2018 年被提出 ${ }^{[3]}$ 。该模型 具有 “采点外推” 的功能, 即, 由已观测地理单元 得到的灾情数据, 推测空白地理单元中的灾情。已 观测单元是采点, 空白单元是信息孤岛。外推的依 据, 是从已观测地理单元总结出的背景数据与灾情 的因果关系 ${ }^{[4]}$ 。

依托互联网进行 “采点外推”，能够及时采集 和处理数据, 并在规定的时间之内推测信息孤岛中 的灾情。基于 “模型-视图-控制器” 框架构建的智 联网, 嵌入空间信息扩散模型后, 能较好地完成这 一任务。任何离线系统, 都完成不了这项任务。

当我们谈及对灾情的快速评估时, 并不限定任 何方法。而本文所指的推测信息孤岛灾情, 限定了 必须依据孤岛外的灾情信息。为使构建的智联网有 良好的拓展性, 本文涉及系统的部分, 常用“评估” 一词替代 “推测”。

推测, 是一种判断各种各样情况的行为, 常见 的有, 纯主观性的层次分析法 ${ }^{[5]}$, 半主观的模糊综 合评价 ${ }^{[6]}$, 更多的则是统计回归 ${ }^{[7]}$ 。地理加权回归, 是一种在空间预测方面很有代表性的线性模型 ${ }^{[8]}[9]$, 也可以拓展为 Logistic 回归和 Poisson 回归 ${ }^{[10]}$ 。然而, 一但凭经验选用的回归函数类型不适合, 预测值就 没有意义。人工神经网络( ANN), 则是不需要人为 假定函数类型，就能识别非线性关系的模型，但是， 如果训练样本由于随机干扰等而出现矛盾冲突, 传 统的 ANN 则不收玫 ${ }^{[11]}$ 。插值, 也是一种常用的推测

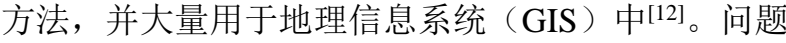
是, 数学插值要求数据具有连续性, 而除了温度场 以外, 大多数地表数据都不满足连续性这一条件。 因此, 对较大地理单元, 数学插值的结果, 精度很 低。

研究表明 ${ }^{[13]}$, 用于推测未观测单元的数据时, 地理空间信息扩散模型比地理加权回归和反向传播 人工神经网络更为有效。因此, 我们选择用地理空 间信息扩散模型来推测信息孤岛中的灾情。

\section{2. 地理空间信息扩散模型}

重大自然灾害发生后, 部分受灾区域由于通信 或道路中断, 形成信息孤岛, 灾情数据无法得到, 应急管理部门很难以对灾区的总体 “灾情” 做出判 断, 人们无法精准调配资源进行及时救助。例如,

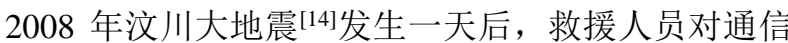
中断的受灾地区，仍然不知其灾情如何。图 1 是重 大自然灾害中的信息孤岛示意图, 一些地理单元上 的死亡人数、重伤人数和灾民人数没有统计上来, 数据不完整。针对此问题, 我们提出地理空间信息 扩散模型 ${ }^{[3]}$, 用已经获得的部分灾情信息, 力求较 精确地推测信息孤岛中的灾情。

不失一般性，假设某一重大自然灾害发生后， 灾区 $G$ 由 $n$ 个地理单元 $g_{1}, g_{2}, \ldots, g_{n}$ 组成, 其中 $q$ 个 地理单元的灾情已知, $n-q$ 个的灾情未知, 且 $n-q$ 远 远小于 $q$ 。灾情已知的地理单元称为已观测单元, 灾情未知的地理单元称为空白单元。也称为灾情信 息孤岛。同时, 我们假定, 可能影响灾情的, 所有 地理单元的自然地理特征、人口、经济状况、自救 能力等背景数据已知。这类与灾情有关且能在灾害 发生前就能收集备用的数据，称为背景数据。

设一个地理单元的背景数据有 $t$ 个分量, 记为

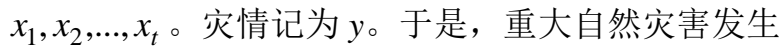
后, 我们能获得一个数据集:

$$
\begin{aligned}
W & =\left\{w_{1}, w_{2}, \cdots, w_{q}\right\} \\
& =\left\{\left(x_{11}, x_{12}, \cdots, x_{1 t}, y_{1}\right), \cdots,\left(x_{q 1}, x_{q 2}, \cdots, x_{q t}, y_{q}\right)\right\}
\end{aligned}
$$




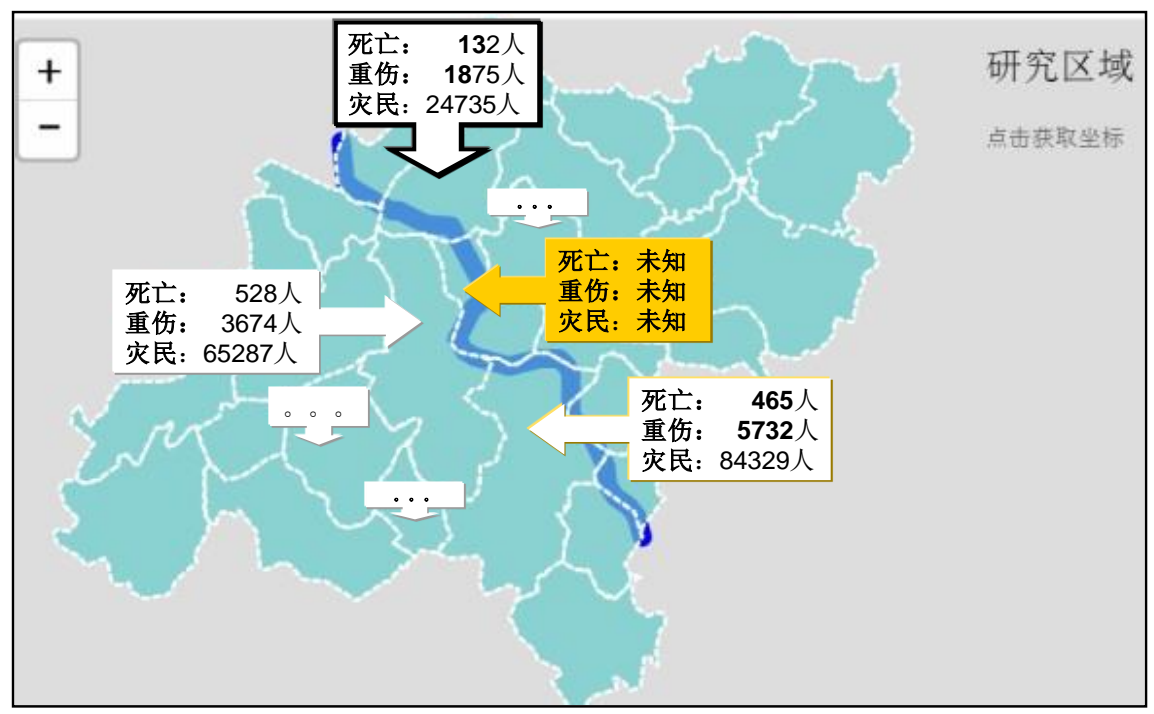

图 1 重大自然灾害中的信息孤岛示意图。未知死亡人数、重伤人数和灾民人数的地理单元是信息孤岛。

式中, $w_{i}=\left(x_{i 1}, x_{i 2}, \ldots, x_{i t}, y_{i}\right), i=1,2, \cdots, q$, 是第 $i$ 个已 观测单元的背景数据和灾情数据。我们称 $W$ 为灾情 学习样本。

一次灾害发生后，除了之前收集到的背景数据， 人们还能通过业务化的自然灾害监测系统，及时获 得描述灾害源属性的一系列数据, 例如, 地震震级、 震源深度和洪水淹没面积等等, 它们通常决定了灾 情的大小。在空间信息扩散模型中, 可用的灾害源 数据一并纳入背景数据中, 作为输入参数进行处理。 灾情, 通常须用多个参数来描述, 例如, 死亡人数、 重伤人数和灾民人数等等, 我们用模型来分别对它 们进行处理。

用空间信息扩散模型推测灾情, 就是用信息扩 散方式从 $W$ 中学习 $y$ 与 $x_{1}, x_{2}, \ldots, x_{t}$ 的模糊关系, 从 而由信息孤岛地理单元的背景数据, 推测灾情。类 似的工作, 由传统的统计回归和人工神经元网络, 都可以做。但多元统计回归的样本须很大, 人工神 经元网络遇到样本点之间不协调时无法收敛。信息 扩散模型则同时解决了这两个问题。

空间信息扩散模型由多维正态扩散、生成模糊 关系和近似推理三部分组成。

\section{1. 多维正态扩散}

将式(1) 中 $W$ 的第 $j$ 个背景数据分量组成的集合记 为 $X_{j}$, 即

$X_{j}=\left\{x_{1 j}, x_{2 j}, \cdots, x_{q j}\right\}, \quad j=1,2, \cdots, t$,

$W$ 中所有灾情组成的集合记为 $Y$, 即

$Y=\left\{y_{1}, y_{2}, \cdots, y_{q}\right\}$.

设 $U_{j}, j=1,2, \ldots, t$, 是分别用于扩散背景数据 $X_{j}$, $j=1,2, \ldots, t$, 的 $t$ 个监控空间 ${ }^{[15]}$, 而 $U_{t+1}$ 是用于扩散灾情
数据 $Y$ 的一个监控空间。令 $\lambda=t+1$, 则背景数据监控 空间和灾情监控空间构成了一个 $\lambda$ 维监控空间:

$U_{1} \times U_{2} \cdots \times U_{\lambda}$.

式中, $U_{j}=\left\{u_{j 1}, u_{j 2}, \cdots, u_{j m_{j}}\right\}, j=1,2, \cdots, \lambda$ 。理论上讲, 监控空间中的监控点个数 $m_{j}$, 可以因 $j$ 的不同而不 同，但为方便起见，通常所有监控空间中的监控点 个数都用同一个数, 并记为 $m$ 。

令 $x_{i \lambda}=y_{i}, i=1,2, \cdots, q$, 式 (1) 中 $W$ 的样本点 $w_{i}=\left(x_{i 1}, x_{i 2}, \cdots, x_{i t}, y_{i}\right)$, 记为 $\lambda$ 维样本点:

$\boldsymbol{x}_{i}=\left(x_{i 1}, x_{i 2}, \cdots, x_{i \lambda}\right) \in W$,

式(4)中的一个 $\lambda$ 维监控点记为,

$\boldsymbol{u}=\left(u_{1 k_{1}}, u_{2 k_{2}}, \cdots, u_{\lambda k_{\lambda}}\right) \in U_{1} \times U_{2} \cdots \times U_{\lambda}$,

（此处 $k_{j} \in\{1,2, \cdots, m\}, j=1,2, \cdots, \lambda ）$, 我们用式(7)的 $\lambda$ 维正态扩散公式, 将 $\boldsymbol{x}_{i}$ 的信息扩散到 $\boldsymbol{u}$.

$\mu\left(\boldsymbol{x}_{i}, \boldsymbol{u}\right)=\prod_{j=1}^{\lambda} \exp \left[-\frac{\left(x_{i j}-u_{j k_{j}}\right)^{2}}{2 h_{j}^{2}}\right]$.

式中的扩散系数 $h_{j}$ 根据式 (1) 中的背景数据和灾情 数据, 分别用式 (8) 进行计算。

$$
h_{j}= \begin{cases}0.8146\left(b_{j}-a_{j}\right), & q=5 ; \\ 0.5690\left(b_{j}-a_{j}\right), & q=6 ; \\ 0.4560\left(b_{j}-a_{j}\right), & q=7 ; \\ 0.3860\left(b_{j}-a_{j}\right), & q=8 ; \quad j=1,2, \cdots, \lambda \\ 0.3362\left(b_{j}-a_{j}\right), & q=9 ; \\ 0.2986\left(b_{j}-a_{j}\right), & q=10 ; \\ 2.6851\left(b_{j}-a_{j}\right) /(q-1), & q \geq 11 .\end{cases}
$$


式中, $b_{j}=\max _{1 \leq i \leq q}\left\{x_{i j}\right\}, a_{j}=\min _{1 \leq i \leq q}\left\{x_{i j}\right\}$.

\section{2. 生成模糊关系}

令

$Q_{k_{1} k_{2} \cdots k_{\lambda}}=\sum_{i=1}^{q} \prod_{j=1}^{\lambda} \exp \left[-\frac{\left(x_{i j}-u_{j k_{j}}\right)^{2}}{2 h_{j}^{2}}\right]$.

我们获得了一个 $U_{1} \times U_{2} \cdots \times U_{\lambda}$ 上的, 由已观测单元 得到的灾情学习样本 $W$ 的信息矩阵, 如式(10)所示:

$$
\begin{aligned}
& \boldsymbol{Q}=\left\{Q_{k_{1} k_{2} \cdots k_{\lambda-1} k_{\lambda}}\right\}_{\underbrace{m \times m \times \cdots \times m}_{\lambda \uparrow}} \\
& \forall k_{\lambda} \in\{1,2, \cdots, m\} \text {, 令 } \\
& S_{k_{\lambda}}=\max _{\substack{1 \leq k_{j} \leq m \\
1 \leq j \leq \lambda-1}}\left\{Q_{k_{1} k_{2} \cdots k_{\lambda-1} k_{\lambda}}\right\} \text {, }
\end{aligned}
$$

和

$r_{k_{1} k_{2} \cdots k_{\lambda-1} k_{\lambda}}=\frac{Q_{k_{1} k_{2} \cdots k_{\lambda-1} k_{\lambda}}}{S_{k_{\lambda}}}$,

我们依据学习样本 $W$, 构造出了背景数据 $x_{1}, x_{2}, \ldots, x_{t}$ 和灾情 $y$ 之间的一个因果型模糊关系矩阵:

$R=\left\{r_{k_{1} k_{2} \cdots k_{\lambda-1} k_{\lambda}}\right\}_{\substack{m \times m \times \cdots \times m \\ \underbrace{}_{\uparrow}}}$

\section{3. 近似推理}

设 $z=\left(z_{1}, z_{2}, \cdots, z_{t}\right)$ 为空白单元的背景数据, 且

$\boldsymbol{u}_{\lambda-1}=\left(u_{1 k_{1}}, u_{2 k_{2}}, \cdots, u_{\lambda-1 k_{\lambda-1}}\right) \in U_{1} \times U_{2} \cdots \times U_{\lambda-1}$.

我们可以用式(14)的 $\lambda-1$ 线性信息分配公式将此 $z$ 变为论域 $U_{1} \times U_{2} \cdots \times U_{\lambda-1}$ 上的一个模糊集, 并取 其隶属度最大值用式(15)进行归一化。

$q_{k_{1} k_{2} \cdots k_{\lambda-1}}=\prod_{j=1}^{\lambda-1} f\left(z_{j}, u_{j k_{j}}\right)$,

式中

$$
\begin{aligned}
& f\left(z_{j}, u_{j k_{j}}\right)=\left\{\begin{array}{l}
1-\frac{\left|z_{j}-u_{j k_{j}}\right|}{u_{j 2}-u_{j 1}},\left|z_{j}-u_{j k_{j}}\right|<u_{j 2}-u_{j 1} \\
0, \text { otherwise }
\end{array}\right. \\
& \left\{\begin{array}{c}
a_{k_{1} k_{2} \cdots k_{\lambda-1}}=\frac{q_{k_{1} k_{2} \cdots k_{\lambda-1}}}{s} \\
s=\max _{\substack{1 \leq k_{j} \leq m \\
1 \leq j \leq \lambda-1}}\left\{q_{k_{1} k_{2} \cdots k_{\lambda-1}}\right\}
\end{array}\right.
\end{aligned}
$$

此模糊集记为 $\tilde{A}$, 其各隶属度值是:

$a_{k_{1} k_{2} \cdots k_{\lambda-1}}, k_{j}=1,2, \cdots, m, j=1,2, \cdots, \lambda-1$.

对于模糊输入 $\tilde{A}$, 使用近似推理公式(16), 我 们可以得到一个具有隶属函数 $\mu_{B}\left(u_{\lambda k_{\lambda}}\right)$ 的模糊输出 $\tilde{B}$ 。

$\mu_{B}\left(u_{\lambda k_{\lambda}}\right)=\max _{\substack{1 \leq k_{j} \leq m \\ 1 \leq j \leq \lambda-1}} \min \left\{a_{k_{1} k_{2} \cdots k_{\lambda-1}}, r_{k_{1} k_{2} \cdots k_{\lambda-1} k_{\lambda}}\right\}$.

最后, 使用式(17)的重心方法, 我们获得了一个 用空白单元背景数据和模糊关系推导出来的灾情推 测值 $\tilde{y}$ :

$\tilde{y}=\frac{\sum_{k_{\lambda}=1}^{m} \mu_{B}\left(u_{\lambda k_{\lambda}}\right) u_{\lambda k_{\lambda}}}{\sum_{k_{\lambda}=1}^{m} \mu_{B}\left(u_{\lambda k_{\lambda}}\right)}$.

需要说明的是, 在推测信息孤岛中灾情时, 我 们使用的是多维线性信息分配公式 ${ }^{[15]}$ 将背景数据转 化为模糊输入，而不是通常空间信息扩散模型中使 用的多维正态信息扩散公式。主要原因是, 当可学 习的样本点只有 10 个或稍多一点的话, 统计规律并 不明显, 用较为粗糙的线性信息分配公式，符合反

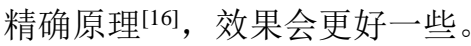

\section{3. 智联网简介}

离线的地理空间信息扩散模型，并不能及时合 理推测信息孤岛灾情。一旦失去时效性, 这一模型 就失去了价值。因为随着时间的推演, 信息孤岛不 复存在, 灾区每一个地理单元上的灾情都会被观测 到。时间，及时救援的宝贵时间，也将失去。

必须依托互联网, 依靠在线采集的数据, 地理 空间信息扩散模型才能及时推测信息孤岛中的灾情。 智联网能为该模型发挥作用提供一个重要的平台, 在线完成这项工作。

由互联网联结多个智能体, 并通过嵌入的模型 集个体小智慧为群体大智慧的网络平台, 称为智联 网[17]。

我们可以将智联网与物联网[18]做一个比较, 说 明智联网的本质所在。物联网是指在物体中嵌入射 频识别等信息传感设备, 通过无线通信技术使得这 些物体与互联网连接起来的一个网络。物联网的主 要功能是实现对物品的自动识别和信息的互联与共 享。无需人的干预, 物联网也可以工作。智联网中, 信息源是人, 而非物。有人提供信息和经验, 数学 模型才能发挥作用, 才能将个人判断的小智慧提升 为群体大智慧, 生成智能产品。因此, 须有人员参 
与, 智联网才可以工作。物联网是一个信息管理系 统，智联网是一个智慧加工和生产系统 ${ }^{[19]}$ 。

\section{1. 智联网的形式化定义}

定义 $1^{[17]}$ 具有观察、演绎、推理和解决问题能 力的个体称为智能体。

人、物等个体均可以作为智能体。例如，在进 行地震灾情的快速评估时, 可以从现场在线提供观 测单元灾情的人员, 是智能体。

定义 $2^{[17]}$ 设 $A$ 是一个智能体集合, $N$ 是 $A$ 使用 的一个网络, $M$ 是处理 $A$ 所提供信息的模型, 三元 体 $\langle A, N, M>$ 称为一个智联网, 记为 $\Phi$ 。

简言之, 智联网是一个由智能体、计算机网络 和信息处理器组成的系统。图 2 展示了最简单的智 联网, 图中的三台计算机 $c_{1}, c_{2}, c_{3}$ 形成网络, 与智能 体 $a_{1}, a_{2}, a_{3}$, 服务器 $S$ 和信息处理模型 $M$ 共同组成智 联网, 其拓扑结构可以抽象为图 3。

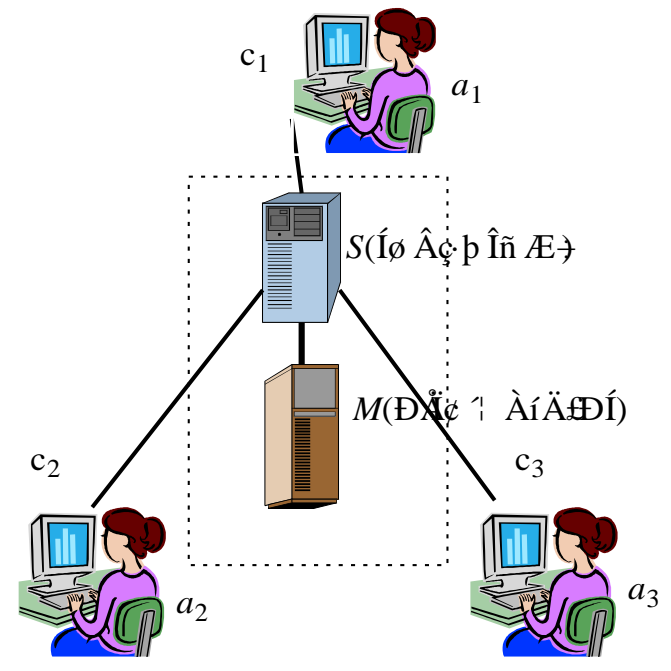

图 2 最简单的智联网 ${ }^{[17]}$

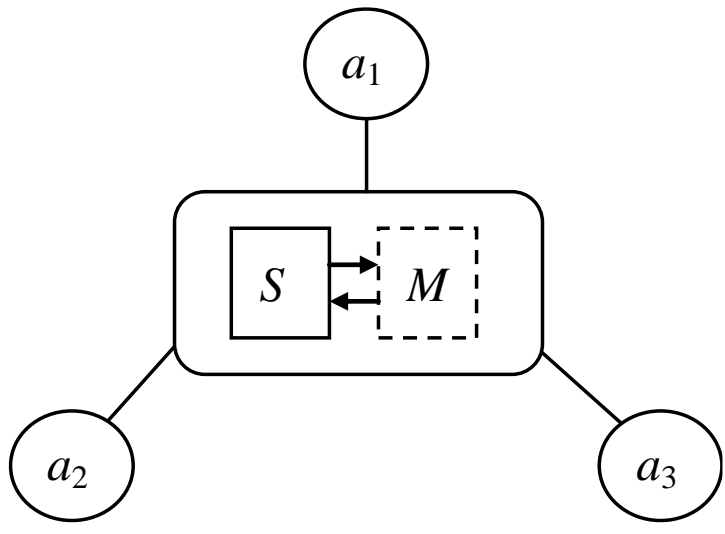

图 3 一个简单智联网的拓扑结构 ${ }^{[20]}$

智联网的工作原理如图 4 所示。智联网用户在 平台内提出问题, 此问题通过网络展示给平台内其 他智能体, 各个智能体对此问题做出自己的解答,
并以多种形式借由网络传输至信息处理模型, 由模 型将这些信息进行综合研判, 形成一个凝聚多源信 息和群体智慧的答案，在线回复给提出问题的用户。 本项研究, 就是将地理空间信息扩散技术作为信息 处理模型嵌入到互联网平台中，使之能在线工作。

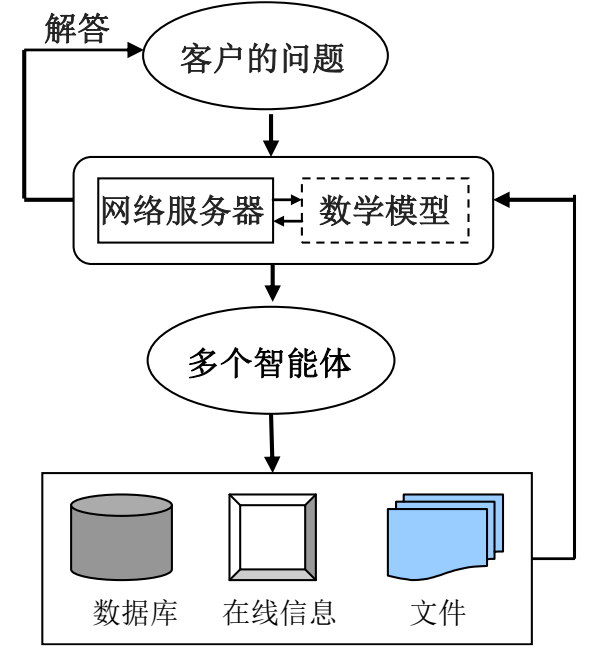

图 4 智联网工作原理 ${ }^{[20]}$

\section{2. 智联网的编程原理}

智联网是一个动态计算机系统, 其基本编程简单原 理如图 5 所示。所谓动态系统是指界面内容会随时 间或用户操作而改变，其基础是有可更新的数据库。 智联网平台使用 MySQL 作为数据库管理系统, 同 时使用服务器端网络编程语言 PHP 来进行数据库操 作和数学计算, 并使用 HTML 等前端语言将数据库 中的信息按照用户要求的方式, 通过终端界面来呈 现给用户。在动态网站中, 除了 PHP 和 HTML 之外, 还会用到 CSS、Javascript 等。

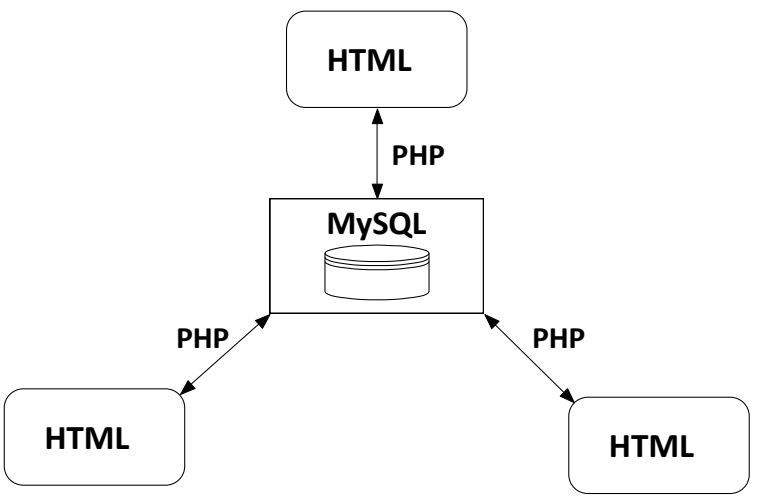

图 5 智联网编程简单原理示意图[19]

本研究不仅仅是将地理空间信息扩散数学模型 嵌入到互联网平台中, 还须根据在线推测灾情的需 求，对推测部分进行系统设计和实现。

\section{4. 在线推测系统整体设计}

用地理空间信息扩散模型推测信息孤岛灾情的 智联网, 按照描述对象的不同, 使用到的相关数据 
分为三类: 一是 “背景数据”, 描述灾区各地理单 元的自然属性和社会属性，例如单元的行政面积、 人口、经济发展水平、建筑结构、地质构造等等; 二是 “灾害源数据”，描述灾害源自身属性，例如 地震震级、震源深度等等; 三是 “灾情数据”，描 述灾害造成的人员、财产、生态损失，例如伤亡人 数、直接经济损失等等。其中, 背景数据在时间维 度上具有较好的稳定性，可在灾害发生前完成收集， 作为灾情推测的基础数据; 灾害源数据只有在灾害 发生时, 才会出现; 而只有灾害发生后且观测过的 地理单元, 才有灾情数据。信息孤岛的灾情, 是经 推测而得。“背景数据” 也称为 “基础数据”,

“灾害源数据” 和 “灾情数据” 均称为 “实时数据” [21]。模型中的输入、输出与基础数据、实时数据的 对应关系如图 6 所示。

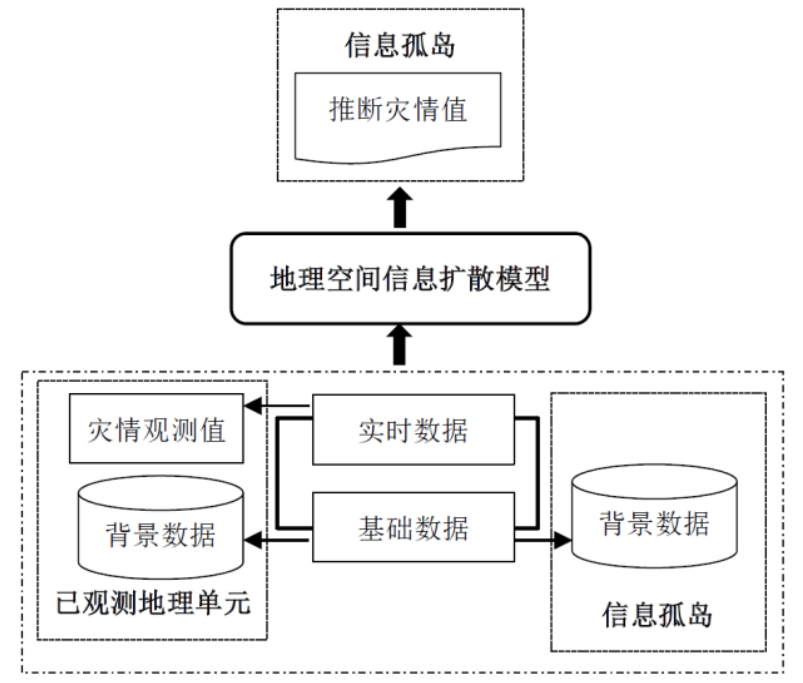

图 6 地理空间信息扩散模型中的数据输入-输出分析

需求分析是开发平台前关键的一项工作, 它是 一个平台能否开发成功的核心因素。推测信息孤岛 灾情的目的是实现救灾阶段空白单元上灾情的快速 推测, 核心功能是收集相关灾害信息并基于地理空 间信息扩散的灾情评估模型进行计算。据此分析, 平台应包括 3 个主要功能 ${ }^{[21]}$ :

（1）灾害信息查询。信息是决策的基础。系统 用户可以通过此功能进行灾害相关信息的查询, 并 依据权限高低有差异性地获取信息。

（2）灾害信息更新。掌握最新灾情进展是救灾 科学性决策的关键。用户通过系统来提交最新灾情 进展, 该部分数据经审核通过后可共享至系统内其 他用户, 并为空白单元的灾情快速推测提供数据支 持。

（3）灾情快速评估。基于有限的已知数据来开 展满足一定精度的空白单元灾情快速评估是现实所 需。灾情快速模块调用系统数据库中的已有数据, 使用人口伤亡率模型、经济损失率模型等进行灾情 评估，为用户的救灾决策提供支撑。
通过对系统进行需求分析，基于面向对象的程 序设计理念，用地理空间信息扩散模型推测信息孤 岛灾情的智联网主要由 6 个模块组成: 前台的数据申 报、数据审核、数据查询、灾情评估及后台的用户 管理、系统管理等。用户角色分为数据申报员、日 常管理员、救灾管理员、系统管理员等四种。不同 的角色享有不同的系统使用权限, 通过调用系统的 不同功能模块来共同完成灾情快速推测的核心任务。

在四种用户角色中，救灾管理员账号由救灾工 作组成员使用, 主要在救灾应急期发挥作用。可以 查看系统内经审核通过的全部数据以及灾情推测结 果。灾害发生后，他们可以查看由数据申报员提交 的 “实时数据申报申请” 并对其进行审核，经审核 通过后的此项数据默认对所有用户公开。救灾管理 者还具有 “灾情快速评估” 功能模块的使用权限, 依次执行指定灾情指标、指定空白单元、选取参与 计算的已观测单元、计算等步骤, 确认推测结果后 可以对其进行保存, 经保存的此项数据仅对救灾管 理者账户用户公开。

图 7 给出了系统功能模块与不同角色的系统使 用权限间的对应关系。其中，灾情推测结果查询仅 对救灾管理员开放, 救灾工作小组可通过媒体、文 件通知等其他方式来向系统用户和其他公众公布最 新的灾情进展。

以推测洪水灾情为例, 系统的整体工作流程可 以用图 8 来表示。当洪水发生后, 作为灾害源的洪 水属性数据和灾情数据不断完善, 数据申报员对实 时数据的更新情况进行持续申报。同时用户可以对 通过审核的基础数据和实时数据, 按照表单和互动 地图相结合的可视化方案进行查看, 掌握最新灾情 进展。救灾管理员一方面需要审核对实时数据更新 的申报, 另一方面可以结合数据的已知情况, 使用 灾情评估模块对信息孤岛中的洪水灾情进行推测。 推测结果因所选取的已观测单元的个数及已观测单 元的组成而异, 当用户确认结果后可以对当下推测 结果进行保存，发布共享给其他救灾管理员。

\section{5. 系统核心功能的实现}

根据上述关于此智联网系统的整体设计思路, 我们着手开发 B/S (Browser/Server, 汶览器/服务 器) 架构的 Web 应用软件, 采用 PHP 作为编程语言, 主要基于 ThinkPHP5（以下简称 “TP5”）框架技术

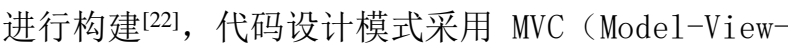
Controller, 模型-视图-控制器）模式, 实现系统 核心计算功能。其他业务模块在现有的技术框架上 加以扩展。

\section{1. 系统整体架构}

$\mathrm{B} / \mathrm{S}$ 架构将系统的核心功能实现集中在服务器 上, 用户无需安装客户端, 只要用户能联网就可以 


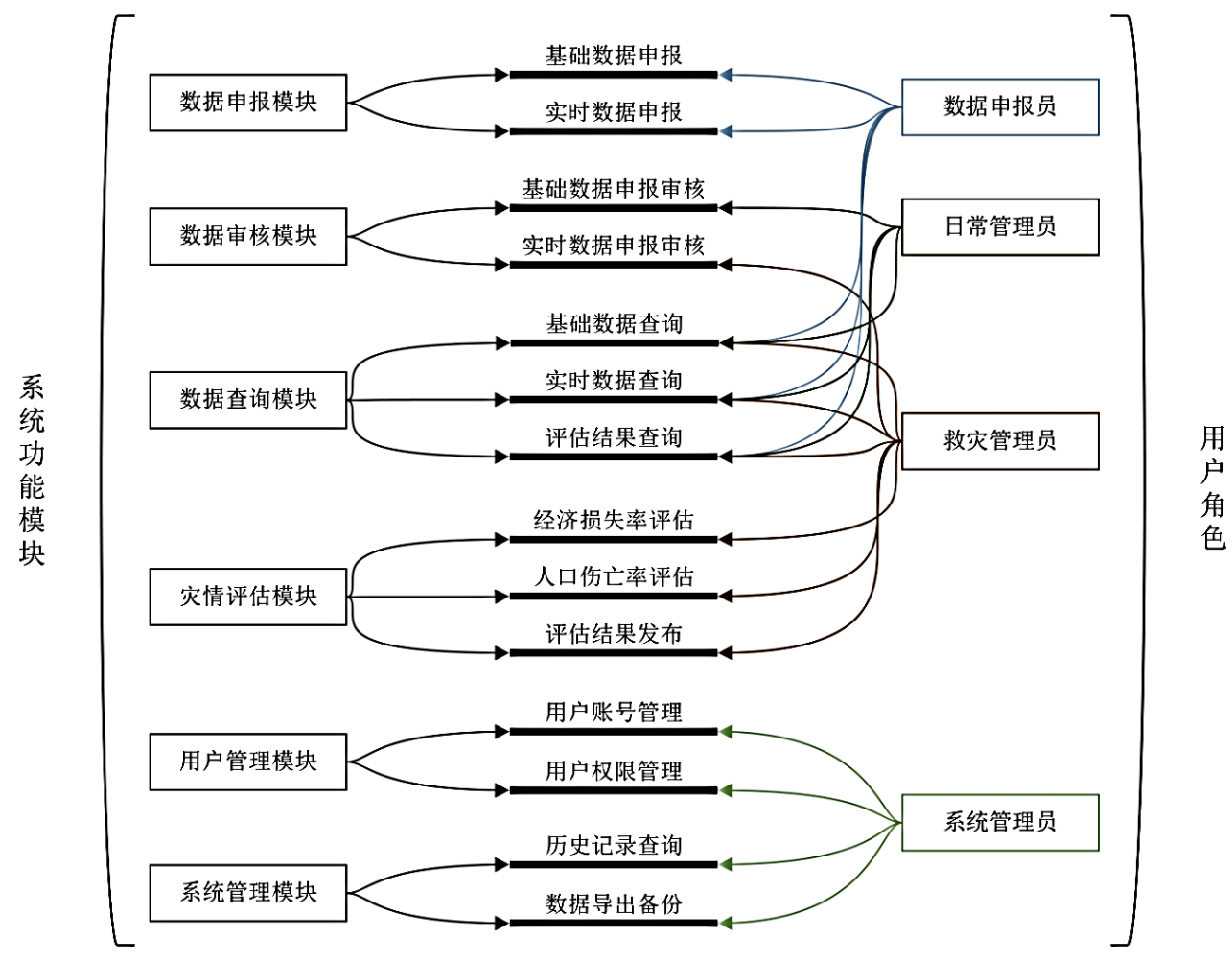

图 7 系统功能模块及用户角色设计 ${ }^{[21]}$ 。

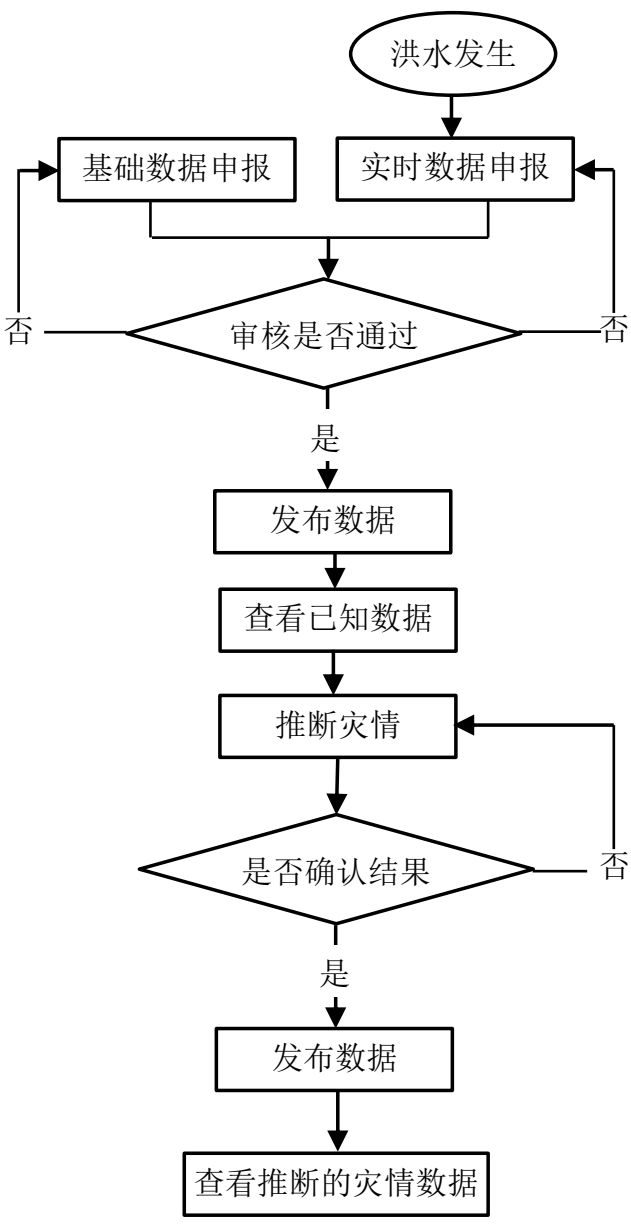

图 8 系统整体工作流程图。
通过浏览器来访问服务端。采用 $\mathrm{B} / \mathrm{S}$ 架构, 用户不 需要对客户端进行升级和维护, 同时开发人员也只 需对服务端的代码进行修改就可以完成系统的统一 维护或升级, 因此基于 $\mathrm{B} / \mathrm{S}$ 架构开发的系统的总成 本较 C/S（Client/Server, 客户机/服务器）架构大 幅度降低。当灾害发生后, 灾害管理者极有可能面 临复杂的环境和有限的硬件设备条件，为了避免客 户端安装限制、适应多终端使用要求，使用 $\mathrm{B} / \mathrm{S}$ 架 构来进行灾情快速评估系统的开发。

MVC 模式的设计思想是把一个应用的输入、处 理和输出流程按照视图 (View)、模型 (Mode1) 和 控制器 (Controller) 分离的方式组织代码, 从而 将一个 Web 应用按照模型层、视图层和控制层的三 层架构进行设计：视图是用户看到的并与之交互的 界面, 向用户显示数据, 同时也直接接收用户请求; 模型是三个部件中的主体部分, 包含业务数据和业 务逻辑; 控制器负责协调整个应用程序的运转。MVC 模式处理流程: 首先用户通过视图即汶览器页面提 交请求, 随后控制器接收浏览器传递来的请求并决 定调用哪个模型来处理此请求; 然后模型进行相应 的业务逻辑处理, 如数据库的增删改查, 并返回处 理结果给控制器; 最后控制器调用相应的视图将处 理结果以合适的方式呈现给用户。

MVC 模式中，模型层、视图层和控制层各司其 职, 在开发期间和后期维护时可以分别对各层进行 改动而互不干扰。应用于模型的代码类似于公共函 数, 因此一个模型能为多个视图提供数据, 而每个 
视图可以从不同角度来表达模型, 优秀的数据可视 化方案可以为用户展现数据的深度价值, 如此也提 高了代码的复用率。当业务流程或规则发生改变而 产生新需求时, 只需要修改模型层; 当需要对网站 的样式进行改变, 只需要修改视图层; 面对用户多 种多样的需求, 控制器可以连接不同的模型和视图 去进行满足。这种特性使得 MVC 模式具有开发效率 高、易于维护、部署便利、利于软件工程化管理等 优点。

\section{2. 系统开发技术与环境}

系统开发技术用到了 PHP 语言、Dreamweavr 编 译器、MySQL 数据库管理系统和 Web 服务器软件 Apache。PHP (Hypertext Preprocessor, 超文本预 处理器）语言用来开发智联网中灾情快速评估 Web 应用程序。PHP 是一种开源的脚本语言。PHP 独特的 语法混合了 C 语言、Java 语言和 Perl 语言以及 PHP 自创的语法, 易于使用且功能强大。语言编译器选 用 Adobe Dreamweavr CS4。该编辑器集网页制作和 管理网站于一体，支持 HTML、CSS、JavaScript、 PHP 等多种网络编程语言, 支持可视化开发。数据 管理使用 MySQL, 是一款关系型数据库管理系统,

采用最常用的结构化查询语言 SQL 进行数据库管理, 支持存储不同类型数据库的数据库表之间的关联关 系以及调用, 效率高、灵活性性强。MySQL 具有开 源、体积小、跨平台性、安全性等特点, 较好地兼 顾了成本与效能。Web 服务器软件使用 Apache, 其 支持跨平台的应用, 能够在几乎所有的主流操作系 统上运行。世界上很多著名的网站如 Amazon、 Yahoo 等都是基于 Apache 进行开发的。

软件开发环境是指在基本硬件和数字软件的基 础上, 为支持系统软件和应用软件的工程化开发和 维护而使用的一组软件。本研究在 WAMP 开发环境下 使用上述技术, 开发出用地理空间信息扩散模型推
测信息孤岛灾情的智联网。

\subsection{ThinkPHP5 框架}

框架，相当于一个房子的骨架 “半成品”，而 组件库相当于各种各样的建筑材料。使用框架开发 的最大优势是高重用性和可扩充性, 项目开发中的 许多基础工作是几乎相同的但占据大量工作量, 而 框架已经将这些基础工作总结成了可以套用的模板, 这使得开发人员得以集中精力在系统的业务逻辑设 计，从而提高效率。同时，框架也可以将复杂的问 题分开来逐个解决，易于控制和分配资源。

ThinkPHP 是一款开源免费的轻量级 PHP 开发框

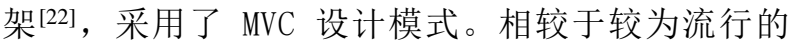
Yii 等其他 PHP 框架, ThinkPHP 更加符合国人的思 维方式和编程习惯。TP5 版本采用了全新的架构思 想，引入了更多的 PHP 新特性，针对 API (Application Programming Interface, 应用程序 接口）开发做了大量优化。

与 TP5 框架开发的智联网后台管理系统相匹配, 我们用 Bootstrap 框架开发客户端使用的 html 网页。 Bootstrap 框架能提供美观、灵活的 HTML5 和 CSS3 规范, 并基于 jQuery 开发了丰富的组件, 覆盖排版、 表格、表单、按钮、图片等 Web 页面设计中常用的 元素, 以及字体图标、下拉菜单、导航、警告框、 弹出框等可复用组件。通过将 Bootstrap 源代码下 载至 TP5 的 public 目录下, 并在 view 文件中的开 头载入 css 样式文件和 js 插件文件即可简便实现在 TP5 中的调用。

\section{4. 技术路径}

系统开发的技术框架如图 9 所示，为实现系统 功能, 本研究在智联网编程原理（图 5) 的基础上 进行了拓展。前端的开发基于 HTML5、CSS3 和

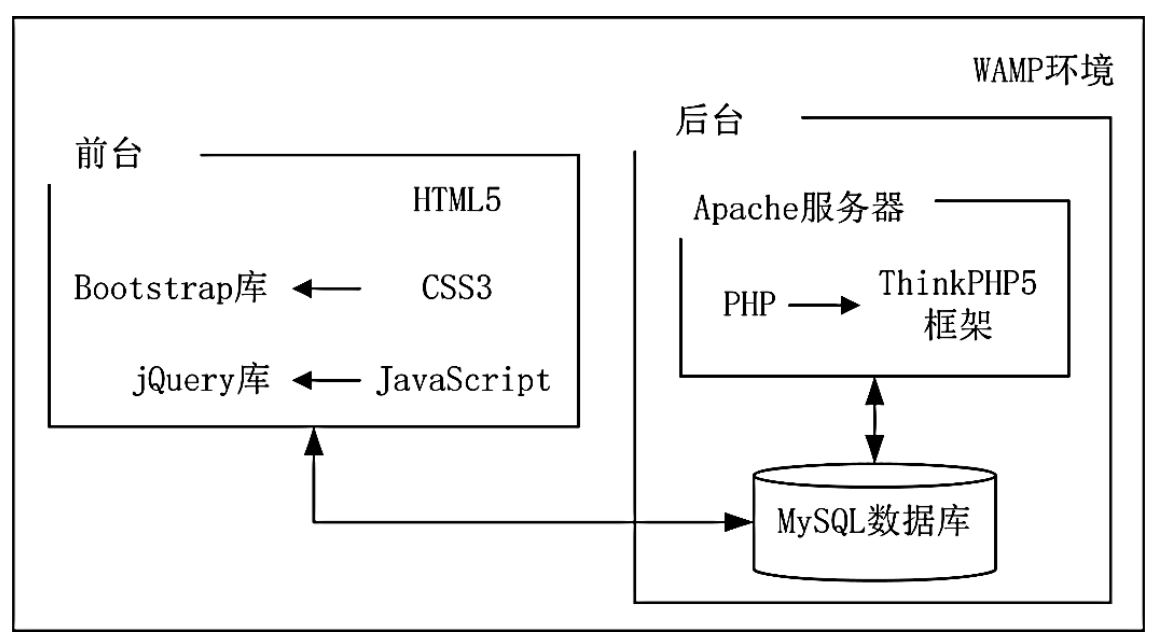

图 9 系统技术框架图。 
JavaScript, 前端即对应于 MVC 模式中的视图层, 为提高交互体验和开发效率，使用 Bootstrap 样式 库中导航栏、下拉菜单、表格等组件。后台服务器 端编程使用 PHP 语言的 TP5 框架, 编写系统的业务 逻辑, 并且实现对 MySQL 的调用。整体项目工程在 WAMP 环境下运行, 使用 Dreamweaver 软件编译代码。

\section{5 核心计算功能的实现}

我们针对大地震后，用智联网进行灾情快速评 估的需要，来实现核心计算功能。

Web 应用程序的开发是一项包含前台开发与后 台开发、需兼顾效率和安全性的复杂工作, 因此首 先对地震灾情快速评估系统中的计算模块进行了编 程, 对系统的核心功能进行实现, 称其为 demo (demonstration, 演示) 版本。为实现本研究所提 模型的计算流程, demo 系统应包括数据录入、数据 查询与执行计算三个基础部分, 其中经济损失评估 和人口伤亡评估, 分别采用式 (18) 和式 (19)。式中 的函数关系 $f$, 由地理空间信息扩散模型构建的因果 关系来表述。

$F_{i}=f\left(I_{i}, S_{i}, X_{i}\right), i=1,2, \cdots, n$

$R_{i}=f\left(I_{i}, S_{i}^{\prime}, P_{i}\right), i=1,2, \cdots, n$

式中, $i-$ 地理单元编号;

$F_{i}$-地理单元上的经济损失率;

$R_{i}$-人口伤亡率;

$I_{i}$-第 $i$ 个地理单元上的平均地震烈度;

$S_{i}$-地理单元面积;

$S_{i}^{\prime}$-地理单元中的建成面积;

$X_{i}$-人均 GDP;

$$
P_{i} \text {-人口密度。 }
$$

建成区面积 $S^{\prime}$ 是衡量地理单元城市化的基本指 标之一, 指地理单元内实际已成片开发建设、市政 公用设施和公共设施基本具备的区域。在一个地理 单元内, 实际分布有建筑的区域是有限的, 而聚集 分布的建筑区人口密度也大, 是地震中伤亡人口的 主要来源区，建成区对应地震致伤高风险区。

\subsection{1. 构建数据库}

数据库设计是动态网站开发的首要工作，合理 的数据库结构设计可以提高应用程序的执行效率, 降低前期开发和后期维护工作的难度。为了方便使 用统计数据, 数据库中的所有地理单元, 均为行政 单元。对应灾情评估模型的输入输出, demo 系统中 所涉数据包含行政单元、基础数据、观测数据和计 算四个主体，用 E-R 图 (Entity Relationship Diagram, 实体-联系图)来表述其逻辑关系，如图 10 所示。

行政单元的属性包括名称和编号两个, 其中编 号为唯一标识。基础数据、实时数据和计算这三个 主体依赖于行政单元而存在, 所以, 它们是相对于 行政单元的弱实体。一个行政单元对应一组基础数 据、一组实时数据, 但对一个行政单元却可以执行 多次计算，计算的时间不同、参与计算的已观测单 元不同会得到不同的灾情评估值。因此，对应着不 同的计算记录。参与计算的已观测单元的组合是不 确定的。实时数据的属性, 例如, 平均烈度、人口 伤亡率观测值、经济损失率观测值等等, 是随着时 间的推移不断完善更新的，因此是可选属性。

\subsection{2. 响应式导航栏}

为实现核心的计算功能, 需实现数据录入、执 行计算、结果查询这三个功能模块，基于此设计系

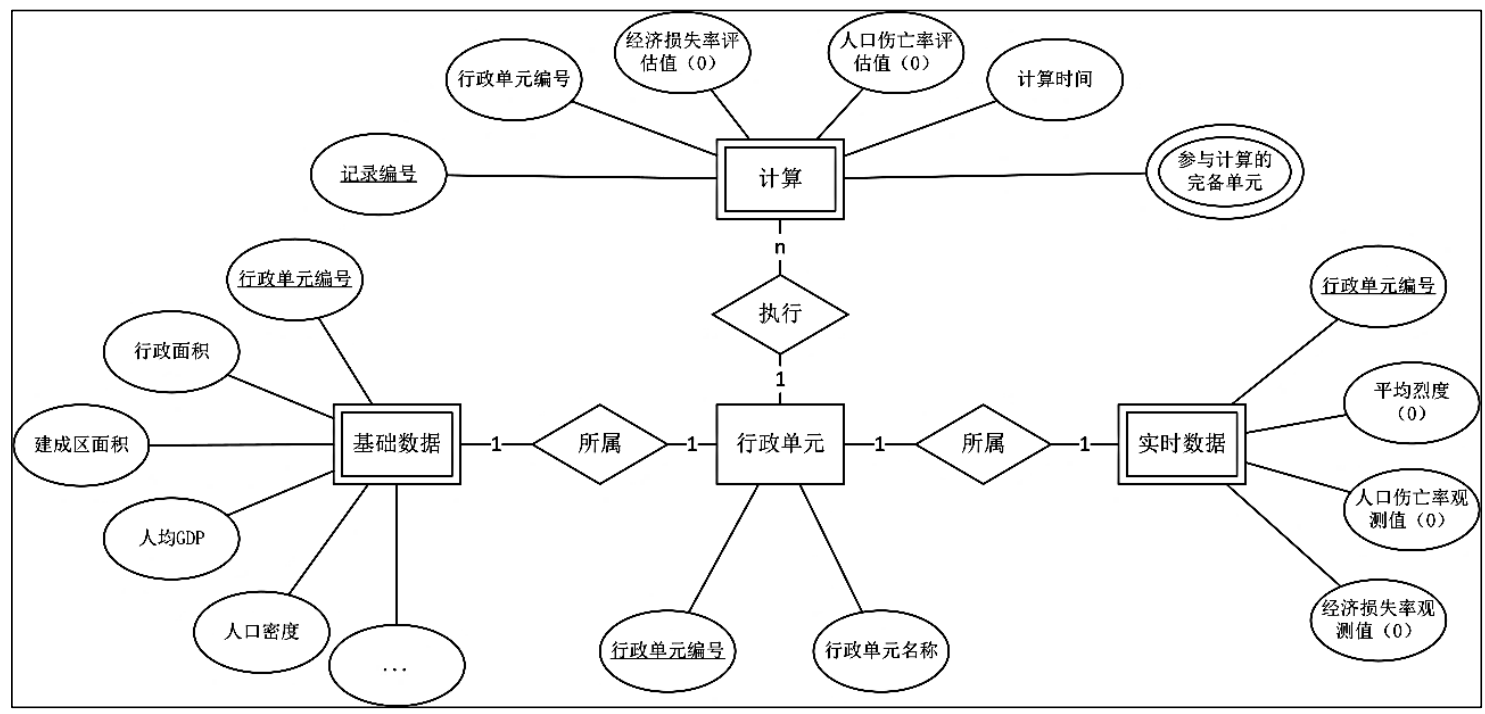

图 10 系统 demo 版数据库 E-R 图。 
统的导航栏结构, 并利用 Bootstrap 工具包实现导 航栏的自适应浏览器布局。当汶览器窗口横向缩小 至一定程度 $(<970 \mathrm{px})$ 时, 导航栏自动缩列成坚排 列表。导航栏含有数据申报、地震灾情评估、灾情 评估结果查询等三个标签, 分别对应三个功能模块, 其中数据申报下拉菜单中包含基础数据申报和实时 数据申报两项, 如图 11 所示。

\section{5 .3 数据录入}

在灾情快速评估系统整体设计中, 将系统的数 据录入分为基础数据录入-日常管理员审核、实时数 据录入-救灾管理员审核两条业务线。demo 版本侧 重打通计算流程, 因此暂且省略数据审核这一环节。 图 12 展示了基础数据申报和实时数据申报页面, 通 过基础数据申报, 用户可以提交研究区行政单元的 行政面积、建成区面积、人口数量、人口密度、GDP 和人均 GDP 等地震发生前就可以收集的数据, 通过 此页面提交的数据会保存至数据库的基础数据表中, 默认最新提交数据会覆盖原有数据; 通过实时数据 申报，用户可以提交灾区行政单元的平均烈度、人 均伤亡率、人均死亡率等随着灾情进展不断更新的 数据, 通过此页面提交的数据会保存至数据库的实
时数据表中, 默认最新提交数据会覆盖原有数据。 用户在汶览器端提交的数据被保存至数据库以自定 义类的方式被写在 controller 文件中。

\subsection{4 地震灾情评估}

掌握灾情相关数据的已知情况是利用地理空间 信息扩散技术进行灾情评估的前提条件，因此在此 页面布置基础数据和实时数据检索查询表单, 方便 用户在了解空白单元和已观测单元分布后有针对性 地利用已知数据进行计算, 页面如图 13 所示。

数据表格默认显示基础数据和实时数据的所有 字段，在右上角设置检索下拉菜单栏，用户可以通 过勾选或取消勾选来查看所需数据。数据表格的显 示和检索查询的实现使用了开源组件 Bootstrap Table, 它扩展了 Bootstrap 的表格操作, 可以便捷 地实现格式化表格、表格选择器、表格工具栏、分 页等样式。

\subsection{5 灾情评估结果查询}

以 2008 年汶川地震的 10 个极重灾区为例，假 设绵竹市的人口伤亡和经济损失都未知, 是空白单 元，而青川县的平均烈度缺失，其余 8 个行政单元

\begin{tabular}{|c|c|c|c|c|c|c|}
\hline (2) 地震灾情快速评估平台 & + & & & - & 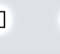 & $x$ \\
\hline$\leftarrow \rightarrow$ C (1) 127.0.0.3 & & & & t5 & $:$ & $\boldsymbol{0}$ \\
\hline \multirow[t]{2}{*}{ 地震灾情快速评估平台 } & 数据申报 - & 地震灾情评估 & 灾情评估结果查询 & & & 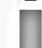 \\
\hline & \multicolumn{2}{|c|}{$\begin{array}{l}\text { 基础数据申报 } \\
\text { 实时数据申报 }\end{array}$} & & & & \\
\hline
\end{tabular}

图 11 导航下拉菜单栏效果图。

\begin{tabular}{|c|c|c|c|}
\hline 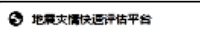 & $x+$ & 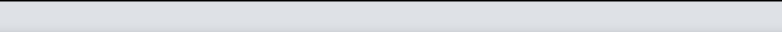 & $-0 \times$ \\
\hline$\leftarrow \rightarrow C \odot$ (127.0.0.3 & & & म $: 0$ \\
\hline 地震灰情快速评估平台 & 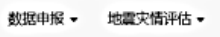 & 灰情评估结果吉询 & \\
\hline 诂选择 & & • & \\
\hline \multicolumn{4}{|l|}{ 行政面积 (虫位：平方公里) } \\
\hline \multicolumn{4}{|c|}{ 建成区面抧（位：平方公里) } \\
\hline \multicolumn{4}{|l|}{ 请输入建成区面积 } \\
\hline \multicolumn{4}{|l|}{ 人口数典 (棵位: 万人) } \\
\hline \multicolumn{4}{|l|}{ 诂输入人口数量 } \\
\hline \multicolumn{4}{|c|}{ 人口密度 (单位: 人平方公里) } \\
\hline \multicolumn{4}{|l|}{ 请输入GDP } \\
\hline \multicolumn{4}{|l|}{ 人均GDP (丑位: 元) } \\
\hline \multicolumn{4}{|l|}{ 请输入人均GDP } \\
\hline 提交申报 & & & \\
\hline
\end{tabular}

图 12 基础数据申报页面 


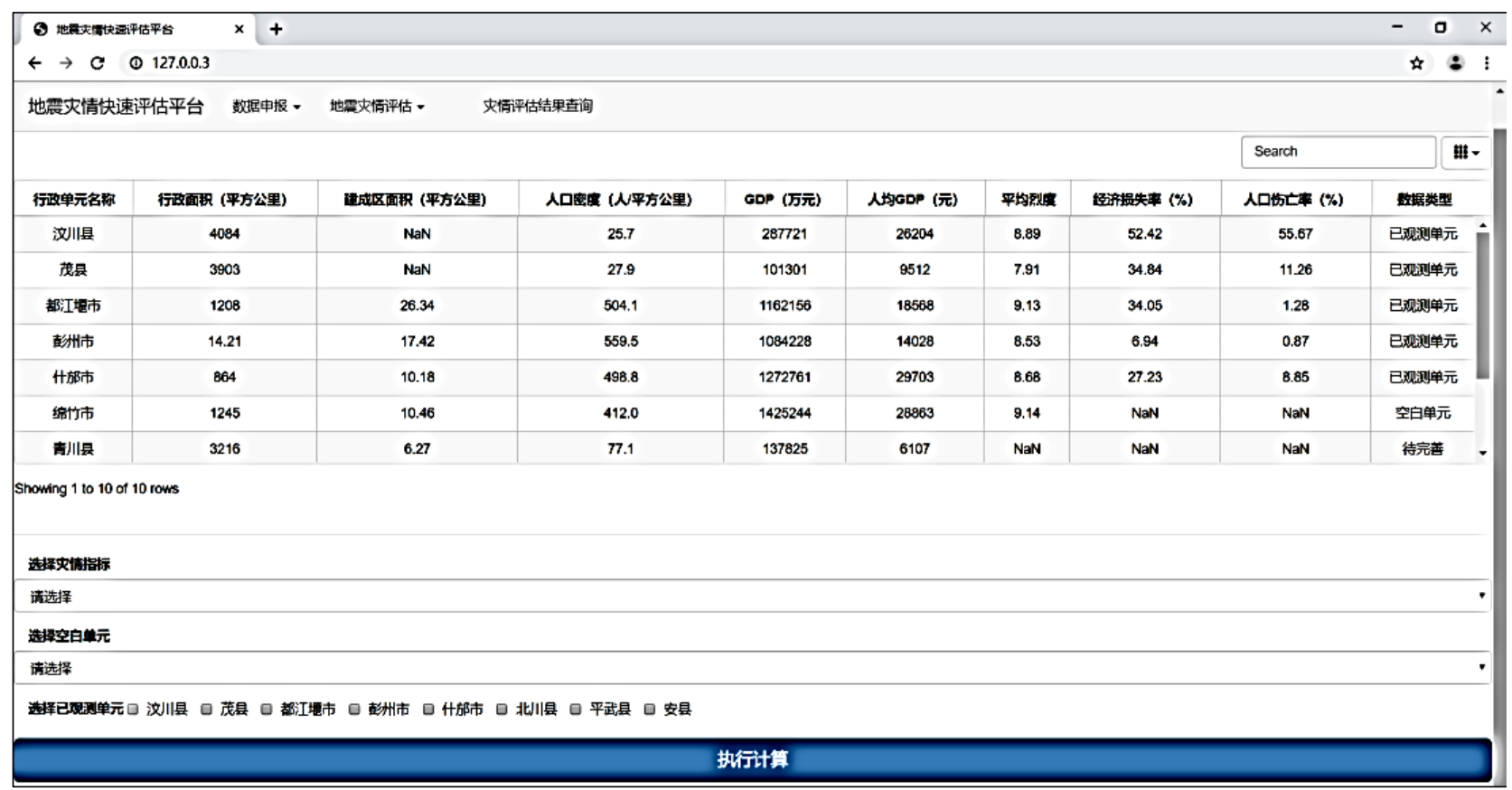

图 13 地震灾情评估页面。

单元是已观测单元, 但其中汶川县和茂县的建成区 面积空缺。分别使用 demo 系统估算绵竹市的人口伤 亡和经济损失，为尽可能提高准确度选取所有可选 取的已观测单元参与计算, 得到的结果在查询页面 展示

使用 TP5 中的 volist 标签进行数据集的输出, 通常模型的 select 方法返回的结果是一个包含字段 名和字段值的二维数组, 可以直接使用 volist 标签 进行输出。

\section{6. 再现推测 2008 年汶川地震灾情}

利用 CuteFTP 软件, 连接远程 ftp 站点, 将在 个人电脑上用开发完成的, 嵌入空间信息扩散模型 的智联网, 上传到此站点的服务器上, 就可以在线 运行此 demo 版本。换言之, 在连接互联网的任何计 算机上输入此智联网的网址, 就能对灾区中信息孤 岛的灾情进行在线 “采点外推”。

我们再现推测 2008 年汶川地震灾情, 来演示其 功能的有效性。智联网系统的主界面如图 14 所示。 2008 年 5 月 12 日 14 时 28 分, 四川省阿坝藏族芫 族自治州汶川县发生 8.0 级大地震, 震中烈度达到 XI 度, 并带来滑坡、崩塌、泥石流、堰塞湖等严重 次生灾害, 给灾区造成了巨大的生命、财产损失和 精神伤害。严重破坏地区超过 10 万平方千米, 包括 极重灾区共 10 个县 (市)。我们选取此 10 个极重 灾区当时得到的数据 (表 1 ), 在智联网上使用地 理空间信息扩散模型, 构建出式 (18)、（19）所需的 因果关系, 再反过来推测这 10 个行政单元的经济损 失率和人口伤亡率, 以检验此智联网的各项功能是 否有效。
将表 1 中的数据录入智联网, 由救灾管理员账 号查看数据、审核, 通过后使用 “灾情快速评估” 功能模块, 推测这 10 个行政单元的经济损失率和人 口伤亡率列入表 2 。

我们采用均方根误差 (Root mean squared error, RMSE）来判断推测值的精度，它由式（20）计算:

$R M S E=\sqrt{\frac{1}{n} \sum_{i=1}^{n}\left(y_{i}-\hat{y}_{i}\right)^{2}}$

式中, $y_{i}, \hat{y}_{i}$ 分别表示观测值和推测值, $n$ 表示行 政单元个数。

列于表 2 的, 用智联网在线上再现推测所得的 2008 年汶川地震灾情, 与用同样的数据和模型, 在 线下计算出来的结果, 完全一致。这说明, 我们所 使用的智联网, 已经具备了, 用地理空间信息扩散 模型，在线上推测信息孤岛灾情的功能。

假定表 1 中的某县（市）其经济损失率和人口 伤亡率数据欠缺, 该县 (市) 就是一座灾情方面的 信息孤岛。此时, 在智联网上学习另 9 个县（市） 的完整资料, 就能推测该信息孤岛的经济损失率和 人口伤亡率, 从而实现 “采点外推” 式的灾情快速 评估, 而不必 “隔空判灾”。

显然, 智联网上推测的灾情精度, 主要受两大 因素制约：一是 “基础数据” 的质量和 “实时数据” 的多少; 二是推测所使用的数学模型。

如果 “基础数据” 欠缺, 推测无法进行。表 2 中没有汶川县和茂县的建成区面积的数据, 嵌入智 


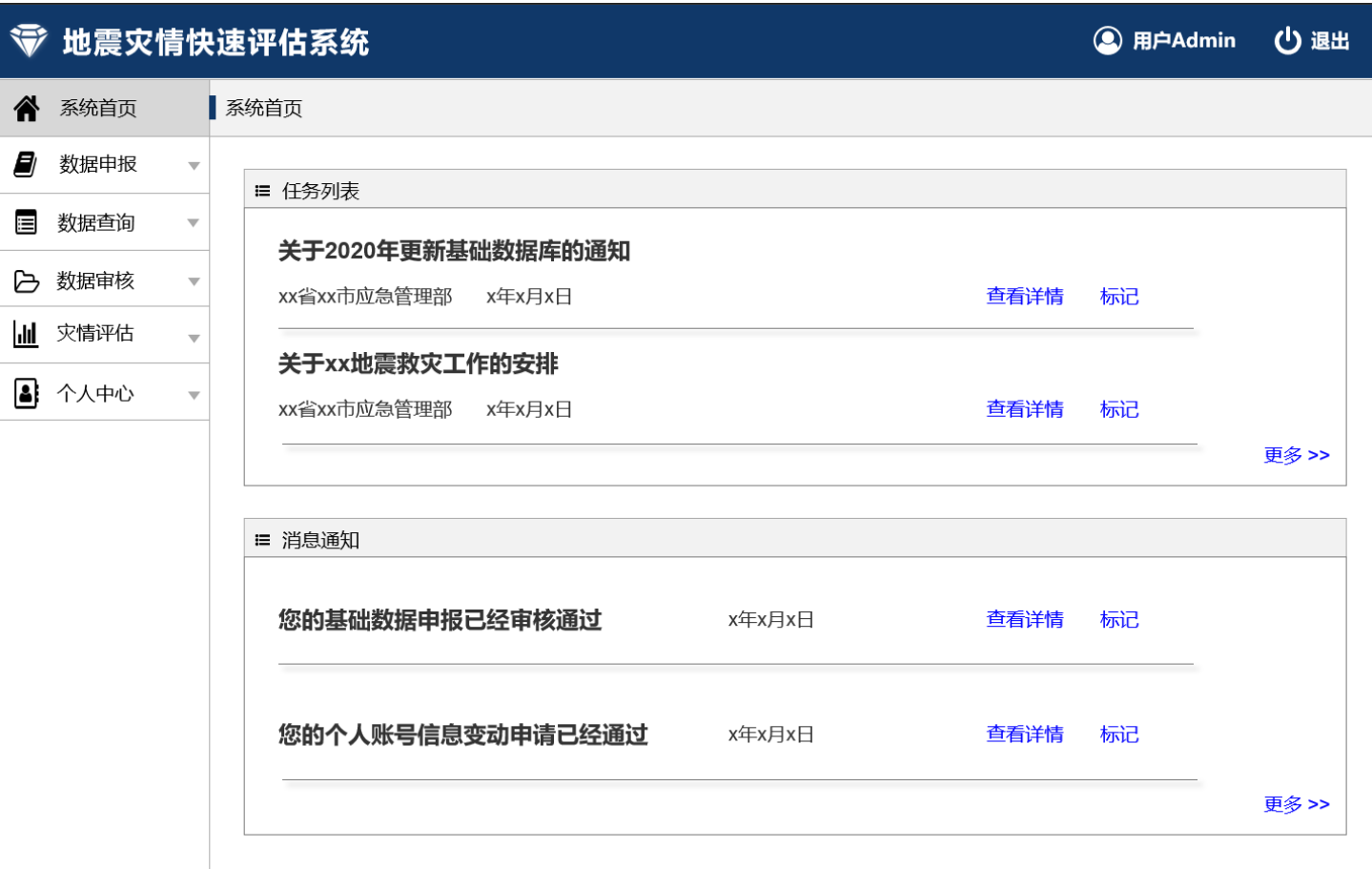

图 14 系统首页页面原型图。

表 1 汶川地震 10 个极重灾区数据表

\begin{tabular}{cccccccccc}
\hline 行政单元 & 行政面积 & 人口数量 & 人口密度 & $\begin{array}{c}\text { 人均 } \\
\mathbf{G D P}\end{array}$ & 平均烈度 & $\begin{array}{c}\text { 死亡人数 } \\
\left(\mathbf{k m}^{2}\right)\end{array}$ & $\begin{array}{c}\text { 人口死 } \\
\text { 亡率 }\end{array}$ & $\begin{array}{c}\text { 经济损 } \\
\text { 失率 }\end{array}$ & $\begin{array}{c}\text { 人口伤 } \\
\text { 亡率 }\end{array}$ \\
\hline 汶川县 & 4084 & 10.5 & 25.7 & 26204 & 8.89 & 23871 & 22.73 & 52.42 & 55.67 \\
茂县 & 3903 & 10.9 & 27.9 & 9512 & 7.91 & 4088 & 3.75 & 34.84 & 11.26 \\
都江堰市 & 1208 & 60.9 & 504.1 & 18568 & 9.13 & 3388 & 0.56 & 34.05 & 1.28 \\
彭州市 & 1421 & 79.5 & 559.5 & 14028 & 8.53 & 1131 & 0.14 & 6.94 & 0.87 \\
什邡市 & 864 & 43.1 & 498.8 & 29703 & 8.68 & 6132 & 1.42 & 27.23 & 8.85 \\
绵竹市 & 1245 & 51.3 & 412.0 & 28863 & 9.14 & 11380 & 2.22 & 26.78 & 9.33 \\
青川县 & 3216 & 24.8 & 77.1 & 6107 & 8.74 & 4819 & 1.94 & 16.91 & 8.17 \\
北川美族 & 2869 & 16 & 55.8 & 8598 & 9.16 & 20047 & 12.53 & 22.74 & 18.59 \\
自治县 & & & & & & & & \\
平武县 & 5974 & 18.7 & 31.3 & 9366 & 8.15 & 6565 & 3.51 & 19.63 & 20.70 \\
安县 & 1404 & 51 & 363.2 & 10434 & 8.89 & 3295 & 0.65 & 3.07 & 3.29 \\
\hline
\end{tabular}

数据来源 : 行政面积、人口数量、GDP、人均 GDP 数据来自 2008 年四川省级、地市级统计年鉴; 死亡人口数据 来自《2008 年汶川地震:灾难剖析》，包含确认死亡人口和失踪人口[23]；平均烈度数据来自史培军等人的研究 ${ }^{[24] 。}$ 人口伤亡率由死亡人数和受伤人数总数除以人口总数计算所得。

联网的式（19）就无法推测人口伤亡率。我国正在

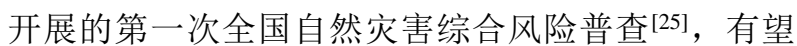
为我国灾害风险的综合管理, 提供较全面的基础数 据, 解决背景数据的问题。
如果 “实时数据” 迟迟无法获取, 就只能从 “采点外推” 退回到 “隔空判灾”。所幸的是, 我 国不仅对主要的自然灾害种类已经建设了大量的监 测系统, 而且 2018 年我国就有了灾害信息员七十余 万名, 实现乡镇网络报灾全覆盖。 
由于地理空间信息扩散模型的普适性，既不需 要人为假设因果关系的函数表述式, 也不涉及所用 参数在地理空间是否连续的问题, 加之有优化处理 小样本的优势，作为智联网中在线自动推测灾情的 数学模型, 效果较理想。

表 2 由智联网推测的灾情

\begin{tabular}{ccclc}
\hline & \multicolumn{2}{c}{$\begin{array}{c}\text { 经济损失率 } \\
\text { 行政单元 }\end{array}$} & $\begin{array}{c}\text { 人口伤亡测值 } \\
\text { 推测值 }\end{array}$ \\
\hline 观测值 & 推测值 \\
茂县 & 52.42 & 49.3 & 55.67 & -- \\
都江堰市 & 34.84 & 33.9 & 11.26 & -- \\
彭州市 & 6.94 & 32.2 & 1.28 & 4.2 \\
什邡市 & 27.23 & 12.8 & 0.87 & 5.7 \\
绵竹市 & 26.78 & 36.0 & 9.85 & 7.6 \\
青川县 & 16.91 & 17.0 & 8.17 & 0.3 \\
北川差族 & 22.74 & 19.1 & 18.59 & 7.2 \\
自治县 & & & \\
平武县 & 19.63 & 31.7 & 20.70 & 0.4 \\
安县 & 3.07 & 15.5 & 3.29 & 9.2 \\
均方根误差 & & 6.990 & & 8.426 \\
\hline
\end{tabular}

注：由于没有汶川县和茂县的建成区面积的数据，此 两行政单元的人口伤亡率无法用式 (19) 推测。

为说明地理空间信息扩散模型在推测精度方面 的优越性, 我们选用两个流行的方法来与它进行比 较: 一是线性回归方法, 最适用于空间统计的是地 理加权回归模型 ${ }^{[8]}$; 二是随机森林方法, 它是一种 能容忍噪声, 由很多决策树分类模型组成的组合分 类模型，是目前数据挖掘、生物信息学中很热门的 非线性建模工具 ${ }^{[27]}$ 。

我们用线性回归和随机森林法分别处理表 1 的 数据, 将它们的均方根误差列入表 3 , 与信息扩散 模型进行比较比。结果表明, 地理空间信息扩散模 型比线性回归方法和随机森林方法的均方根误差都 明显地小，说明推测的灾情更为准确。

表 3 均方根误差对比

\begin{tabular}{c|cc}
\hline & 经济损失率误差 & 人口伤亡率误差 \\
\hline 信息扩散 & 6.990 & 8.426 \\
线性回归 & 16.901 & 16.022 \\
随机森林 & 14.627 & 7.198 \\
\hline
\end{tabular}

\section{7. 结论与讨论}

在人人都是传感器的网络时代, 对重大自然灾 害的 “隔空判灾”, 越来越显得过于粗糙。精准救 助的时代要求, 对 “采点外推” 寄予期望。大量自 然灾害监测系统的存在、全国自然灾害综合风险普
查将建设的数据库, 以及灾害信息员乡镇网络报灾 全覆盖, 都为我国依托互联网对灾情进行准确度更 高的快速评估打下了坚实的基础。

用地理空间信息扩散模型推测信息孤岛灾情的 智联网 demo 版本的在线运行成功, 是继“城市道路 暴雨内涝风险分析与预警智联网》[28]和“社区安全风 险雷达智联网, ”[29]两个专用智联网后的又一个成功案 例, 并且在设计思路和开发技术上有所改进, 适用 面更广。

由于受传统思维惯性的影响，国内原创的 “智 联网”一直没有大量的资源投入，很难像泊来的 “物联网”一样快速发展。加之“唯论文、唯职称、 唯学历、唯奖项” 的考核指挥棒, 急功近利左右大 量科研资源的走向，“智联网” 等原创科技的生存 空间很小。所以，直到今天，尚无一个商业化智联 网问世。作为国家重点研发计划的一部分, 本项研 究完成的这一智联网平台, 须在软件工程方面得到 进一步的完善, 才能最终进入业务化系统，任重而 道远。

\section{致谢}

本项目受国家重点研发计划课题 “重大自然灾 害多层级精准救助关键技术研究”（编号: 2017YFC1502902）资助。本文中智联网的实现，由 作者指导的研究生尹怡然在读期间完成。由于其就 职单位工作性质的缘故, 不便在本文中署名。作者 对她在本文中的贡献，表示衷心感谢！

\section{参考文献}

[1] 王晓青, 丁香, 王龙等, 四川汶川 8 级大地震灾害 损失快速评估研究，地震学报，2009,31(2):205211. DOI: https://doi.org/10.3321/j.issn:02533782. 2009. 02.010

[2] 安基文, 徐敬海, 聂高众, 白仙富, 高精度承灾 体数据支撑的地震灾情快速评估, 地震地质, 2015, 37(4):1225-1241. DOI: https://doi.org/10. 3969/j.issn.0253-4967.2015.04.022

[3] 黄崇福, 地理空间上的信息扩散及其在风险分析 中的应用,一带一路背景下的风险分析和危机反 应, 黄崇福, 王成军编(中国灾害防御协会风险分 析专业委员会第八届年会论文集, 西安, 2018 年 10 月 20-21 日), Atlantis Press, Paris, 2018, pp.1-7. DOI: https://doi.org/10.2991/rac-18.2018.1

[4] 黄崇福, 借助背景数据的地理空间信息扩散技术, Journal of Risk Analysis and Crisis Response, 2019,9(1):2-10. DOI: https://doi.org/10.1007/9783-642-21455-4_3

[5] J. Chen, W. Li, W.K. Chen, S.P. Zhang, Assessment of earthquake prevention and disaster reduction capability of county - level administrative units in Gansu Province, Journal of Risk Analysis and Crisis Response, 2017, 7(4): 
214 - 224. DOI: https://doi.org/10.2991/jrarc.2017. 7.4 .3

[6] X.F. Cheng, H.H. Sun, Z. Yuan, G.L. Xu, Flood disaster risk assessment and spatial distribution characteristics along the Yangtze River in Anhui Province, Journal of Risk Analysis and Crisis Response , 2014 , 4(4): 238 - 242. DOI: https://doi.org/10.2991/jrarc.2014.4.4.6

[7] Y. Ding, M. Mu. Research on the development of county finance in Guizhou Province in the promotion of precise poverty alleviation, Journal of Risk Analysis and Crisis Response, 2018 , 8(1):52 - 60. DOI: https://doi.org/10.2991/jrarc.20 18.8.1.6

[8] C. Brunsdon, A.S. Fotheringham, M.E. Charlton, Geographically weighted regression: a method for exploring spatial nonstationarity, Geographical Analysis, 1996, 28(4): 281-298. DOI: https://doi. org/10.1111/j.1538-4632.1996.tb00936.x

[9] D.J. Lieske, D. J. Bender, A robust test of spatial predictive models: geographic cross-validation, Journal of Environmental Informatics, 2011, 17(2): 91-101. DOI: https://doi. org/10.3808/jei.20110019 1

[10] A.S. Fotheringham, C. Brunsdon, M. Charlton, Geographically Weighted Regression: the Analysis of Spatially Varying Relationships, Chichester, USA: John Wiley \& Sons, 2002.

[11] C.F. Huang, C. Moraga, A diffusion-neuralnetwork for learning from small samples, International Journal of Approximate Reasoning, 2004, 35: 37-161. DOI: https://doi.org/10.1016/j. ijar.2003.06.001

[12] K.A. Eldrandaly, M.S. Abu-Zaid, Comparison of six GIS-based spatial interpolation methods for estimating air temperature in western Saudi Arabia. Journal of Environmental Informatics, 2011, 18(1), 38 - 45. DOI: https://doi.org/10.3808/jei.2011001 97

[13] C.F. Huang, Two judging criteria to check validity of a model for filling gaps caused by incomplete geospatial data, Environmental Research, 2020, 186: 109401. DOI: https://doi.org/10.1016/j.envres. 2020.109401

[14] 张勇, 许力生, 陈运泰, 2008 年汶川大地震震源机 制的时空变化, 地球物理学报, 2009, 52 (2): 379389.

[15] 黄崇福,自然灾害风险分析与管理, 科学出版社, 北京, 2012 年.

[16] 李金锋, 黄崇福, 宗恬, 反精确现象与形式化研 究, 系统工程理论与实践, 2005, 25(4):128132.DOI:https://doi.org/10.3321/j.issn:10006788.2005.04.021

[17] 黄崇福, 风险分析在线服务的智联网, Journal of Risk Analysis and Crisis Response, 2011,
1(2):110-117. DOI: https://doi.org/10.2991/ijcis. 2011.1.2.4

[18] L.D. Xu, W. He, S. Li, Internet of things in industries: a survey, IEEE Transactions on Industrial Informatics, 2014, 10(4):2233-2243. DOI:10.1109/TII.2014.2300753

[19] 黄崇福, 智联网原理及其核心技术的发展, Journal of Risk Analysis and Crisis Response, 2017, 7(3):146-155. DOI:https://doi.org/10.2991/ jrarc. 2017.7.3.5

[20] C.F. Huang, Multiple internet of intelligences for risk analysis, Journal of Risk Analysis and Crisis Response, 2014, 4(2): 61-71.DOI: https://doi.org /10.2991/jrarc.2014.4.2.2

[21] 尹怡然，基于地理空间信息扩散技术的空白单 元地震灾情快速评估模型研究及系统设计, 北 京师范大学学位论文, 2020.

[22] 李秀珍, 基于 MVC 的 ThinkPHP5 框架研究, 现 代信息科技，2020，(14):90-92. DOI: https://doi.org/10.19850/j.cnki.2096-4706.2020.14. 027

[23] Y. Chen, D.C. Booth, 《2008 年汶川地震:灾难剖 析》第一章:汶川地震，世界地震译从，2019, 50(3): 266-302. DOI: https://doi.org/10.16738/j.cnki.issn.10033238.201903005

[24] 史培军, 灾害风险科学, 北京师范大学出版社, 北 京, 2016.

[25] 汪明, 全国自然灾害综合风险普查工程（二）, 开展第一次全国自然灾害综合风险普查的重要 意义, 中国减灾, 2020, (10月上): 24-27.

[26] D.J. Lieskeand, D. J. Bender, A robust test of spatial predictive models: geographic crossvalidation. Journal of Environmental Informatics, 2011, 17(2):91-101. DOI: https://doi.org/10.3808/ jei.201100191

[27] 方匡南, 吴见涁, 朱建平, 谢邦昌, 随机森林方法 研究综述, 统计与信息论坛, 2011, 26(3):32-38. DOI: https://doi.org/10.3969/j.issn.10073116.2011. 03.006

[28] 曾凡雷, 郭树军, 黄崇福, 城市道路暴雨内涝风险 分析与预警智联网服务系统及方法，中国发明专 利, ZL 201710514431.3 .2020 年 6 月 29 日.

[29] C.F. Huang, T. Wu, O. Renn, A risk radar driven by internet of intelligences serving for emergency management in community, Environmental Research, 2016, 148: 550-559. DOI: https://doi.org/10.1016/j.envres.2016.03.016 